\title{
The effects of third-party transfers in sequential anchored bargaining
}

\author{
Suchan Chae $^{1}$ (D) Seho Kim $^{2}$
}

Accepted: 16 December 2018 / Published online: 26 December 2018

(c) The Author(s) 2018

\begin{abstract}
We analyze a bargaining game where an anchor player bargains sequentially with $n$ non-anchor players over the division of a pie in the presence of third-party transfers and show that there exists a unique perfect equilibrium. A lump-sum transfer is jointly shared by all players, while a transfer proportional to a player's share affects only the party that has to make that transfer. When lump-sum transfers are zero, the anchor player and each non-anchor player bargain as if there is no further bargaining. It turns out that the anchor player and the last non-anchor player are in the most disadvantageous position with our bargaining protocol.
\end{abstract}

Keywords Sequential bargaining - Anchored bargaining · Tax incidence

JEL Classification C78 · C22 · C72

\section{Introduction}

An $n$-person pure bargaining problem deals with a situation where multiple parties split a pie with a unanimous agreement. The outcome depends on the bargaining procedure used.

Rubinstein (1982) introduced a bargaining game where two players make alternating proposals on the partition of a pie in possibly infinite rounds until they reach an agreement. The costly time lapse between bargaining rounds provides an incentive for players to come to an agreement. He shows that there exists a unique perfect equi-

Suchan Chae

chae2@kaist.ac.kr

Seho Kim

shkim33@econ.umd.edu

1 College of Business, Korea Advanced Institute of Science and Technology, Daejeon 34141, South Korea

2 Department of Economics, University of Maryland, College Park 20742, MD, USA 
librium for the game. The equilibrium outcome approaches the Nash (1950, 1953) solution as the time lapse approaches zero. It has been well known [see, for instance, Shaked's argument in Sutton (1986) and Herrero (1985)] that Rubinstein's result may not obtain in $n$ ( $\geq 3$ )-person extensions.

Jun (1987) and Chae and Yang (1988, 1994) introduced an extension of Rubinstein's game to a multi-person bargaining situation and showed that there exists a unique subgame perfect equilibrium. In their $n$-person games, a player proposes the share to another player. If the second player accepts the proposal, the game essentially becomes an $(n-1)$-person game. If the responding player rejects the proposal, the game becomes another $n$-person bargaining with a permutation of players. ${ }^{1}$

In many real-life bargaining situations, there is a fixed player who simultaneously or sequentially bargains with other players. Let us call such a player an anchor player. Some works in the literature are motivated by Coase's railroad-farmer problem, where a railroad company, an anchor player, has to obtain permission from farmers to undertake a project. ${ }^{2}$ Among them, Cai (2000) analyzes a bargaining game where the order of non-anchor players is fixed, but the order of their agreements with the anchor player can be endogenous. Since every non-anchor player wants to hold up the bargaining process in the hope of getting a larger share, delay can arise in equilibrium. Xiao (2018) analyzes a game where an anchor player chooses the order of non-anchor players and shows that the anchor player will choose non-anchor players with smaller disagreement payoffs first. Lee and Liu (2013) analyze a game where a non-anchor player demands his share based on the reputation of the anchor player. If an anchor player rejects the offer, a random exogenous payment is made by the anchor player to the non-anchor player. They show that an anchor player may reject even an attractive offer to build up reputation. Genicot and Ray (2006) analyze a model where a principal offers contracts to some agents, and the payoffs of agents without contracts decrease as the number of such agents decreases. Iaryczower and Oliveros (2017) analyze a model where one or two principals make offers to a probabilistically determined pool of agents. They show that the agents can be better off with a single principal than with two competing principals when there are positive externalities among agents.

In this paper, we analyze a bargaining game where an anchor player bargains sequentially with $n$ non-anchor players over the division of a pie in the presence of third-party transfers. One key characteristic of the protocol is that the anchor player can move to the next stage to bargain with the next non-anchor player only if the anchor player reaches an agreement with the current opponent. In a two-person Rubinstein-type alternating-proposal bargaining game, the outcome is determined by balancing the bargainers' impatience, measured by delay premium, which is the amount a player is willing to give up in order to avoid delay. Rubinstein's existence result depends critically on the assumption that the delay premium is an increasing function of the size of the stake. We show that there exists a unique perfect equilibrium for our model if the delay premium is a linear function of the size of the stake. The protocol is the only one we are aware of that yields a unique perfect equilibrium for any discount

\footnotetext{
${ }^{1}$ Krishna and Serrano (1996) obtains a similar result for a variation of the model where if $k$ players accept a proposal then the game is essentially reduced to an $(n-k)$-person game.

2 See Coase (1960).
} 
factors with an outcome which is different from that of Chae and Yang $(1988,1994)$ for an $n$-person game.

The protocol can be applied to situations where there is a hierarchy among nonanchor player. The protocol is not fair to every participant. It turns out that in this setup the anchor player and the last non-anchor player are in the most disadvantageous position, for the anchor player has to reach an agreement with the non-anchor player at each stage before it can move to the next non-anchor player. Among the nonanchor players, earlier players in the hierarchy have advantages. The protocol may be applicable to the following kinds of situations:

Example 1 In order to implement a real-estate development project, the developer has to first secure the land from the landowner, then find a construction company to build structures, and then find people who want to occupy the structures built. What is left of the value of the project becomes the developer's profit.

Example 2 An estate has to be split among a group of hierarchical claimants. A lawyer negotiates with all claimants and will keep what is left of the estate after securing agreements from all claimants.

Example 3 When a firm goes bankrupt, the remaining value of its assets has to be distributed among the creditors, typically with a preset order of claims. An equity holder takes some portion of the remaining value in many real world bankruptcy cases. $^{3}$ Such an equity holder may have taken the role of an anchor player in our model.

In all of the above examples and in most real-life situations, claimants need to pay taxes to the government and intermediation fees to intermediaries out of their revenues. Thus, in our model, we will assume that a player has to make a transfer to a third party if an agreement is reached. The transfer can be negative as in the case of a subsidy. The transfer can be lump-sum or proportional to a player's gain. We will study a general case that allows both lump-sum and proportional transfers. We show that in equilibrium a lump-sum transfer is jointly shared by all players, but a proportional transfer affects only the player that has to make that transfer. In particular, if lump-sum transfers are zero, at each stage the anchor player and each non-anchor player bargain as if there is no further bargaining.

\section{A bargaining model with third-party transfers}

Players $P_{0}, P_{1}, \ldots, P_{n}$ bargain over the split $\left(s_{0}, s_{1}, \ldots, s_{n}\right)$ of a pie $\pi$ so that $s_{0}+$ $s_{1} \cdots+s_{n}=\pi$. When all players reach an agreement, $P_{i}$ receives payoff $x_{i}(\geq 0)$ after making some transfer to a third party. The third party could be the government, in which case the transfer would be a tax, or an investor, in which case the transfer would be a return on the investment. The transfer could be lump-sum or proportional to what a player receives from bargaining. We will consider a transfer scheme that encompasses both the lump-sum and proportional cases: $x_{i}=\left(1-\tau_{i}\right)\left(s_{i}-T_{i}\right)$, where

${ }^{3}$ See, for instance, Bharath, Panchapegesan, and Werner (2010). 
$\tau_{i}<1$ and $T_{i} \in \mathbb{R}$. If $\tau_{i}<0$ or $T_{i}<0, P_{i}$ actually receives some payment from a third party. It should be noted here that $T_{i}$ is different from the usual breakdown payoff in that it affects a player's payoff only if an agreement is reached.

If the agreement is reached at time $t$, the outcome is $\left(x_{0}, x_{1}, \ldots, x_{n} ; t\right)$. We will only work with the case where payoffs are nonnegative. $P_{i}$ has preferences over payoff-time pairs $\left(x_{i}, t\right) \in \mathbb{R}_{+}^{2}$. Assume that the preferences are represented by a transitive and complete relation $\preccurlyeq_{i}$ (with the usual convention for $\prec_{i}$ and $\sim_{i}$ ) which satisfies the following axioms:

A1 (Continuity): If $\left(x, t_{1}\right) \prec_{i}\left(y, t_{2}\right)$, then there exist open neighborhoods of $A$ and $B$ of $\left(x, t_{1}\right)$ and $\left(y, t_{2}\right)$, respectively, such that $\left(\tilde{x}, \tilde{t}_{1}\right) \prec_{i}\left(\tilde{y}, \tilde{t}_{2}\right)$ for any $\left(\tilde{x}, \tilde{t}_{1}\right) \in A$ and $\left(\tilde{y}, \tilde{t}_{2}\right) \in B$.

A2 (Desirability): For any $t$, if $x<y$ then $(x, t) \prec_{i}(y, t)$.

A3 (Impatience): If $x>0$ and $t_{1}<t_{2}$, then $\left(x, t_{1}\right) \succ_{i}\left(x, t_{2}\right)$.

A4 (Stationarity): If $\left(x, t_{1}\right) \sim_{i}\left(y, t_{2}\right)$ then $\left(x, t_{1}+r\right) \sim_{i}\left(y, t_{2}+r\right)$ for any $r$.

A5 (Neuter): $\left(0, t_{1}\right) \sim_{i}\left(0, t_{2}\right)$ for any $t_{1}, t_{2}$.

For any $t \geqslant 0$ and $x \geqslant 0$, let $(x, t) \sim_{i}\left(p_{i}(x, t), 0\right)$. Then $p_{i}(x, t)$ represents player $i$ 's subjective present value of payoff $x$ received at $t$. Impatience can be measured by the delay premium $x-p_{i}(x, t)$. Chae and Yang (1994) introduces an $n$-person bargaining solution that generalizes Rubinstein's (1982) solution as one that equalizes the delay premium across players. For such a solution to exist, another axiom is required:

A6 (Monotonicity of Delay Premium): $x-p_{i}(x, t)$ increases in $x$ for any $t>0$.

For the main result of this paper, we will focus on preferences that satisfy a stronger axiom:

A6* (Linearity of Present Value): $p_{i}(x, t)=\rho_{i}{ }^{t} x$ for some $\rho_{i}$ such that $0<\rho_{i}<1$.

If the present value is linear in $x$, the delay premium is also linear in $x$ :

$$
x-p_{i}(x, t)=\left(1-\rho_{i}^{t}\right) x .
$$

Fishburn and Rubinstein (1982) show that a preference relation that satisfies axioms A $1-\mathrm{A} 5$ can be represented by a utility function of the form $U_{i}(x, t)=\delta_{i}{ }^{t} u_{i}(x)$, where $0<\delta_{i}<1$. With this utility representation, one has

$$
p_{i}(x, t)=u_{i}^{-1}\left(\delta_{i}^{t} u_{i}(x)\right),
$$

and the linearity of the present value means ${ }^{4}$

$$
u_{i}(x)=x^{a_{i}} \quad \text { for some } a_{i}>0,
$$

for then

$$
p_{i}(x, t)=\rho_{i}{ }^{t} x,
$$

where

$$
\rho_{i}=\delta_{i}^{\frac{1}{a_{i}}}
$$

\footnotetext{
4 See Appendix for the proof of utility representation.
} 
Note that there can be multiple combinations of utility parameters $\delta_{i}$ and $a_{i}$ that yield the same preference parameter $\rho_{i} .^{5}$

We will consider a bargaining protocol where there exists an anchor player who engages with each of the other players in a sequence of bilateral bargaining. Thus we define a bargaining game $G\left(\pi, t, P_{0} ; P_{1}, \ldots, P_{n}\right)$ with total pie $\pi\left(\geq T_{0}+\sum_{i=1}^{n} T_{i}\right)$, starting period $t(=0,1, \ldots, \infty)^{6}$, an anchor player $P_{0}$ and $n$ non-anchor players $P_{1}, \ldots, P_{n}$ recursively as follows:

If $n=0$, the game $G\left(\pi, t, P_{0} ; P_{1}, \ldots, P_{n}\right)$ is a trivial one-person game where $P_{0}$ receives the payoff $x_{0}=\left(1-\tau_{0}\right)\left(\pi-T_{0}\right)$ in period $t$. Let $n \geq 1$. If $\pi=$ $T_{0}+\sum_{i=1}^{n} T_{i}, G\left(\pi, t, P_{0} ; P_{1}, \ldots, P_{n}\right)$ is a trivial game where players receive payoffs $(0,0, \ldots, 0)$ in period $t$. Let $\pi>T_{0}+\sum_{i=1}^{n} T_{i}$. In period $t, P_{0}$ offers $P_{1}$ 's share $s_{1}$ $\in\left[T_{1}, \pi-\left(T_{0}+\sum_{i=2}^{n} T_{i}\right)\right]$ to $P_{1}$. If $P_{1}$ accepts the offer, then the remaining game becomes $G\left(\pi-s_{1}, t, P_{0} ; P_{2}, \ldots, P_{n}\right)$, where $\pi-s_{1} \geq T_{0}+\sum_{i=2}^{n} T_{i}$. If $P_{1}$ rejects the offer, then the game moves to the period $t+1$, and $P_{1}$ proposes his own share $s_{1}$ $\in\left[T_{1}, \pi-\left(T_{0}+\sum_{i=2}^{n} T_{i}\right)\right]$ to $P_{0}{ }^{7}$ If $P_{0}$ accepts $P_{1}$ 's demand, then the remaining game becomes $G\left(\pi-s_{1}, t+1, P_{0} ; P_{2}, \ldots, P_{n}\right)$. If $P_{0}$ rejects $P_{1}$ 's demand, then the remaining game becomes $G\left(\pi, t+2, P_{0} ; P_{1}, \ldots, P_{n}\right)$. This bargaining procedure continues recursively until only the anchor player remains, that is, until agreements are reached between the anchor player and all non-anchor players. If bargaining does not end in finite periods, no player receives any payment. This outcome is denoted $(0, \infty)$, and we assume that $(0, t) \sim_{i}(0, \infty)$ for any $t$, for any $P_{i}$. The game $G\left(\pi, t, P_{0} ; P_{1}\right)$ is a standard Rubinstein game with two players $P_{0}$ and $P_{1}$, where player $P_{0}$ is the initial proposer, if $T_{0}=T_{1}=0$ and $\tau_{0}=\tau_{1}=0$.

Put

$$
\begin{aligned}
r_{i} & =\frac{1-\rho_{i}}{1-\rho_{0} \rho_{i}}, \\
\hat{r}_{i} & =\frac{1-\rho_{0}}{1-\rho_{0} \rho_{i}}
\end{aligned}
$$

for $i=1, \ldots, n$.

Theorem 1 Suppose that the intertemporal preferences of the players $P_{0}, P_{1}, \ldots, P_{n}$ satisfy $A 1-A 5$ and $A 6^{*}$. The n-person game $G\left(\pi, t, P_{0} ; P_{1}, \ldots, P_{n}\right)$ has a unique perfect equilibrium with the outcome $\left(x_{0}, x_{1}, \ldots, x_{n} ; t\right)$, where

$$
x_{0}=\left(1-\tau_{0}\right)\left\{\pi-\left(T_{0}+\sum_{j=1}^{n} T_{j}\right)\right\} \prod_{j=1}^{n} r_{j}
$$

\footnotetext{
5 Thus the essential preference parameter is $\rho_{i}$, not $\delta_{i}$ and $a_{i}$.

6 Each period $t$ is regarded as a discrete time point $t$.

7 Chae and Yang (1992) analyze a bargaining process with alternating demands.
} 
and

$$
x_{i}=\left(1-\tau_{i}\right)\left\{\pi-\left(T_{0}+\sum_{j=1}^{n} T_{j}\right)\right\}\left(\prod_{j=1}^{i-1} r_{j}\right)\left(1-r_{i}\right) \text { for } i=1, \ldots, n \text {. }
$$

Proof We will use mathematical induction. The theorem is trivially true for $n=0 .{ }^{8}$ Suppose that the theorem is true for $n=k-1(\geq 0)$. We will show that the theorem is true for $n=k$. If $P_{0}$ and $P_{1}$ reach an agreement at time $t^{\prime}$ to split the pie as $\left(s_{0}, s_{1}\right)$, where $s_{0} \geq T_{0}+\sum_{j=2}^{k} T_{j}, s_{1} \geq T_{1}$, and $s_{0}+s_{1}=\pi$, then the remaining subgame becomes a $k$-person game $G\left(s_{0}, t^{\prime}, P_{0} ; P_{2}, \ldots, P_{k}\right)$. Since the theorem is true for $n=k-1$, we know that in the unique perfect equilibrium of this $k$-person subgame, the agreement is reached at time $t^{\prime}$ and $P_{0}$ 's payoff is

$$
\left(1-\tau_{0}\right)\left\{s_{0}-\left(T_{0}+\sum_{i=2}^{k} T_{i}\right)\right\} \prod_{j=2}^{k} r_{j} \equiv \phi_{0}\left(s_{0}\right) .
$$

Thus we can replace this subgame by the outcome $\left(\phi\left(s_{0}\right), \psi\left(s_{1}\right), t^{\prime}\right)$, where

$$
\psi\left(s_{1}\right)=\left(1-\tau_{1}\right)\left(s_{1}-T_{1}\right)
$$

to obtain a two-person game between $P_{0}$ and $P_{1}$. In this modified game, $P_{0}$ and $P_{1}$ bargain over shares $\left(s_{0}, s_{1}\right)$ such that $s_{0}+s_{1}=\pi$ and $s_{1} \in\left[T_{1}, \pi-\left(T_{0}+\sum_{i=2}^{k} T_{i}\right)\right]$. Consider an equivalent game, where $P_{0}$ and $P_{1}$ bargain over normalized shares $\left(\sigma_{0}, \sigma_{1}\right)$ such that $\sigma_{0}+\sigma_{1}=1$ and $\sigma_{1} \in[0,1]$, where

$$
\begin{gathered}
\sigma_{0}=\frac{s_{0}-\left(T_{0}+\sum_{j=2}^{k} T_{j}\right)}{\pi-\left(T_{0}+\sum_{j=1}^{k} T_{j}\right)} \equiv f\left(s_{0}\right), \\
\sigma_{1}=\frac{s_{1}-T_{1}}{\pi-\left(T_{0}+\sum_{j=1}^{k} T_{j}\right)} \equiv g\left(s_{1}\right) .
\end{gathered}
$$

Put

$$
\begin{aligned}
& \alpha\left(\sigma_{0}\right)=\phi\left(f^{-1}\left(\sigma_{0}\right)\right)=\sigma_{0}\left(1-\tau_{0}\right)\left\{\pi-\left(T_{0}+\sum_{j=1}^{k} T_{j}\right)\right\} \prod_{j=2}^{k} r_{j}, \\
& \beta\left(\sigma_{1}\right)=\psi\left(g^{-1}\left(\sigma_{0}\right)\right)=\sigma_{1}\left(1-\tau_{1}\right)\left\{\pi-\left(T_{0}+\sum_{j=1}^{k} T_{j}\right)\right\} .
\end{aligned}
$$

\footnotetext{
${ }^{8}$ It is a standard convention that $\sum_{j=1}^{0} T_{i}=0$ and $\prod_{j=1}^{0} r_{j}=1$.
} 
Define preferences $\prec_{0}^{\alpha}$ over $\left(\sigma_{0}, t\right) \in[0,1] \times \mathbb{R}_{+}$by

$$
\left(\sigma_{0}, t\right) \prec_{0}^{\alpha}\left(\sigma_{0}^{\prime}, t^{\prime}\right) \text { if and only if }\left(\alpha\left(\sigma_{0}\right), t\right) \prec_{0}\left(\alpha\left(\sigma_{0}^{\prime}\right), t^{\prime}\right) \text {, }
$$

and preferences $\prec_{1}^{\beta}$ over $\left(\sigma_{1}, t\right) \in[0,1] \times \mathbb{R}_{+}$by

$$
\left(\sigma_{1}, t\right) \prec_{1}^{\beta}\left(\sigma_{1}^{\prime}, t^{\prime}\right) \text { if and only if }\left(\beta\left(\sigma_{1}\right), t\right) \prec_{1}\left(\beta\left(\sigma_{1}^{\prime}\right), t^{\prime}\right) \text {. }
$$

It is obvious that $\prec_{0}^{\alpha}$ and $\prec_{1}^{\beta}$ satisfy axioms A1-A5. We will now show that they also satisfy axiom A6. For $P_{0}$, the present value $p_{0}^{\alpha}\left(\sigma_{0}, t\right)$ of his normalized share is defined from

$$
\left(\sigma_{0}, t\right) \sim_{0}^{\alpha}\left(p_{0}^{\alpha}\left(\sigma_{0}, t\right), 0\right),
$$

i.e,

$$
\left(\alpha\left(\sigma_{0}\right), t\right) \sim_{0}\left(\alpha\left(p_{0}^{\alpha}\left(\sigma_{0}, t\right)\right), 0\right) .
$$

Since $\left(\alpha\left(\sigma_{0}\right), 1\right) \sim_{0}\left(\alpha\left(p_{0}^{\alpha}\left(\sigma_{0}, 1\right)\right), 0\right)$, one has

$$
\left.\alpha\left(p_{0}^{\alpha}\left(\sigma_{0}, 1\right)\right)=p_{0}\left(\alpha\left(\sigma_{0}\right), 1\right)\right)
$$

i.e.,

$$
p_{0}^{\alpha}\left(\sigma_{0}, 1\right)=\alpha^{-1}\left(\rho_{0} \alpha\left(\sigma_{0}\right)\right)=\rho_{0} \sigma_{0} .
$$

Similarly, one has

$$
p_{1}^{\beta}\left(\sigma_{1}, 1\right)=\rho_{1} \sigma_{1} .
$$

Thus the preferences $\prec_{0}^{\alpha}$ and $\prec_{1}^{\beta}$ satisfy axiom A6 (indeed, A6*). From Rubinstein (1982), it follows that there exists a unique perfect equilibrium with the outcome $\left(\sigma_{0}, \sigma_{1} ; 0\right)=\left(v, p_{1}^{\beta}(w, 1) ; 0\right)$, where $v$ and $w$ satisfy the equation system

$$
\begin{aligned}
& v+p_{1}^{\beta}(w, 1)=1 \\
& p_{0}^{\alpha}(v, 1)+w=1,
\end{aligned}
$$

i.e.,

$$
\begin{aligned}
& v+\rho_{1} w=1, \\
& \rho_{0} v+w=1 .
\end{aligned}
$$

One has

$$
\begin{aligned}
v & =r_{1}, \\
w & =\hat{r}_{1},
\end{aligned}
$$


and thus

$$
\begin{aligned}
& x_{0}=\alpha(v)=\left(1-\tau_{0}\right)\left\{\pi-\left(T_{0}+\sum_{j=1}^{k} T_{j}\right)\right\} \prod_{j=1}^{k} r_{j} \\
& x_{1}=\beta\left(p_{1}^{\beta}(w, 1)\right)=\left(1-\tau_{1}\right)\left\{\pi-\left(T_{0}+\sum_{j=1}^{k} T_{j}\right)\right\}\left(1-r_{1}\right)
\end{aligned}
$$

From the induction hypothesis, one also has

$$
\begin{aligned}
x_{i} & =\left(1-\tau_{i}\right)\left\{f^{-1}\left(r_{1}\right)-\left(T_{0}+\sum_{i=2}^{k} T_{i}\right)\right\}\left(\prod_{j=2}^{i-1} r_{j}\right)\left(1-r_{i}\right) \\
& =\left(1-\tau_{i}\right)\left\{\pi-\left(T_{0}+\sum_{i=1}^{k} T_{i}\right)\right\}\left(\prod_{j=1}^{i-1} r_{j}\right)\left(1-r_{i}\right)
\end{aligned}
$$

for $i=2, \ldots, k$.

For a structural understanding of the theorem, let us now look at the theorem from the standpoint of an arbitrary time point during the bargaining process, taking the risk that some arguments in the proof are replicated here. Consider a situation where the anchor player $P_{0}$ has already reached agreement with non-anchor players $P_{1}, \ldots, P_{i-1}$ for their shares $s_{1}, \ldots, s_{i-1}$. Let $v_{i-1}=\pi-\left(s_{1}+\cdots+s_{i-1}\right)(\geq$ $\left.T_{0}+\sum_{j=i}^{n} T_{j}\right)$ be the residual pie. The remaining game is an $(n-i+2)$-person game $G\left(v_{i-1}, t, P_{0} ; P_{i}, \ldots, P_{n}\right)$. In this game, $P_{0}$ initially bargains with $P_{i}$, taking into account the effect of reaching agreement for $P_{i}$ 's share $s_{i} \in\left[T_{i}, v_{i-1}-\left(T_{0}+\right.\right.$ $\left.\left.\sum_{j=i+1}^{n} T_{j}\right)\right]$ on the remaining game $G\left(v_{i}, t, P_{0} ; P_{i+1}, \ldots, P_{n}\right)$, where $v_{i}=v_{i-1}-s_{i}$. In other words, in order to bargain with $P_{i}$, the anchor player $P_{0}$ needs to know the outcome of $G\left(v_{i}, t, P_{0} ; P_{i+1}, \ldots, P_{n}\right)$.

By backward mathematical induction, the anchor player $P_{0}$ realizes that this remaining game will be resolved immediately with the outcome $\left(x_{0}, x_{i+1}, \ldots, x_{n} ; t\right)$, where

$$
x_{0}=\left(1-\tau_{0}\right)\left\{v_{i}-\left(T_{0}+\sum_{j=i+1}^{n} T_{j}\right)\right\} \prod_{j=i+1}^{n} r_{j} \equiv \phi_{i}\left(v_{i}\right) \text {. }
$$

Thus from the standpoint of $P_{0}$ and $P_{i}$, the $(n-i+1)$-person subgames $G\left(v_{i-1}-\right.$ $\left.s_{i}, t, P_{0} ; P_{i+1}, \ldots, P_{n}\right)$ for all possible values of $s_{i} \in\left[T_{i}, v_{i-1}-\left(T_{0}+\sum_{j=i+1}^{n} T_{j}\right)\right]$ can be replaced by their outcomes $\left(\phi_{i}\left(v_{i-1}-s_{i}\right), \psi_{i}\left(s_{i}\right), t\right)$, where

$$
\psi_{i}\left(s_{i}\right)=\left(1-\tau_{i}\right)\left(s_{i}-T_{i}\right)
$$

resulting in a two-person game $\hat{G}\left(v_{i-1}, t, P_{0} ; P_{i}\right)$. 
Solving the game $\hat{G}\left(v_{i-1}, t, P_{0} ; P_{i}\right)$, where two players $P_{0}$ and $P_{i}$ split the pie $v_{i-1}$ into $\left(s_{0}, s_{i}\right)$ such that $s_{0}+s_{i}=v_{i-1}$ and $s_{i} \in\left[T_{i}, v_{i-1}-\left(T_{0}+\sum_{j=i+1}^{n} T_{j}\right)\right]$ is exactly the same as solving the game $\hat{G}\left(\pi, t, P_{0} ; P_{1}\right)$ in the proof of the theorem when we substitute $v_{i-1}=\pi$ and $i=1$. Let $v_{i}$ be $P_{0}$ 's share in the perfect equilibrium of the two-person game $\hat{G}\left(v_{i-1}, t, P_{0} ; P_{i}\right)$ and $w_{i}$ be $P_{i}$ 's share in the perfect equilibrium of a two-person game which is the same as $\hat{G}\left(v_{i-1}, t, P_{0} ; P_{i}\right)$ except that $P_{i}$ makes the first proposal. Then, with obvious notation for the present value functions $p_{0}^{\phi_{i}}\left(s_{0}, t\right)$ and $p_{i}^{\psi_{i}}\left(s_{i}, t\right)$ for shares $s_{0}$ and $s_{i}$, the perfect equilibrium outcome of the two-person game $\hat{G}\left(v_{i-1}, t, P_{0} ; P_{i}\right)$ is $\left(s_{0}, s_{i} ; t\right)=\left(v_{i}, p_{i}^{\psi_{i}}\left(w_{i}, 1\right) ; t\right)$, where $v_{i}$ and $w_{i}$ satisfy the equation system

$$
\begin{gathered}
v_{i}+p_{i}^{\psi_{i}}\left(w_{i}, 1\right)=v_{i-1}, \\
p_{0}^{\phi_{i}}\left(v_{i}, 1\right)+w_{i}=v_{i-1} .
\end{gathered}
$$

In particular, the solution equalizes the delay premium between the two players:

$$
v_{i}-p_{0}^{\phi_{i}}\left(v_{i}, 1\right)=w_{i}-p_{i}^{\psi_{i}}\left(w_{i}, 1\right)
$$

i.e.,

$$
\left(1-\rho_{0}\right)\left(v_{i}-T_{0}-\sum_{j=i+1}^{n} T_{j}\right)=\left(1-\rho_{i}\right)\left(w_{i}-T_{i}\right) .
$$

If we compare $P_{0}$ 's delay premium in the $i$-th stage of the $(n+1)$-person game $G\left(\pi, t, P_{0} ; P_{1}, \ldots, P_{n}\right)$,

$$
\left(1-\rho_{0}\right)\left\{v_{i}-\left(T_{0}+\sum_{j=i+1}^{n} T_{j}\right)\right\},
$$

with $P_{0}$ 's delay premium in the standard two-person game $G\left(v_{i-1}, t, P_{0} ; P_{i}\right)$ game,

$$
\left(1-\rho_{0}\right)\left(v_{i}-T_{0}\right),
$$

the only difference is $T_{0}+\sum_{j=i+1}^{n} T_{j} v s . T_{0}$. When $P_{0}$ and $P_{i}$ bargain taking into account of the fact that $P_{0}$ has to further bargain with $P_{i+1}, \ldots, P_{n}$, it effectively changes $P_{0}$ 's lump-sum transfer from $T_{0}$ to $T_{0}+\sum_{j=i+1}^{n} T_{j}$.

If $\sum_{j=i+1}^{n} T_{j}=0$, then the shares of $P_{0}$ and $P_{i}$ in the $i$-th stage of the $(n+1)$-person game $G\left(\pi, t, P_{0} ; P_{1}, \ldots, P_{n}\right)$ coincides with the unique subgame perfect equilibrium agreement for the two-person game $G\left(v_{i-1}, t, P_{0} ; P_{i}\right)$. That the anchor player $P_{0}$ has to further bargain with subsequent players $P_{i+1}, \ldots, P_{n}$ over the split of $s_{0}=v_{i-1}$ has no influence on $P_{0}$ 's bargaining with $P_{i} .{ }^{9}$ If $\sum_{j=i+1}^{n} T_{j} \neq 0$, however, the outcome of the

\footnotetext{
9 This is evident from the expression for $x_{i}$ (in Theorem 1), which includes $T_{j}$ and $r_{j}$ only for $j=0,1, \ldots, i$ when $\sum_{j=i+1}^{n} T_{j}=0$.
} 
bargaining between $P_{0}$ and $P_{i}$ depends on the transfers $T_{i+1}, \ldots, T_{n}$ of $P_{i+1}, \ldots, P_{n}$. If $\sum_{j=i+1}^{n} T_{j}>0$, the existence of subsequent non-anchor players $P_{i+1}, \ldots, P_{n}$ hurts the current non-anchor player $P_{i}$. If, however, $\sum_{j=i+1}^{n} T_{j}<0$, then $P_{i}$ actually benefits from their existence.

It is interesting to note that the players bargain as if there is a well-defined net surplus to be divided among all players, i.e., $\pi-\left(T_{0}+\sum_{j=1}^{n} T_{j}\right)$, even though the players do not bargain simultaneously but sequentially. In particular, lump-sum transfers are fully shared by all players. This property is most likely due to our assumption that intertemporal preferences are such that present value is linear in the size of the stake. It is certainly convenient for our analysis but not necessarily a general principle to rely on.

The theorem also shows that in contrast to a lump-sum transfer, which is shared jointly by all players, a proportional transfer affects only the player that has to make that transfer. Consider the case where the lump-sum transfers are zero $\left(T_{0}=T_{1}=\cdots=\right.$ $T_{n}=0$ ) and the proportional transfer is actually a proportional tax. In this case, the burden of the tax falls completely on the taxed party. Chae (2002) studies tax incidence with a Nash solution, which is the limit of the outcome of a two-person Rubinstein bargaining model. He shows that the burden of a tax falls completely on the taxed party, if the taxed party has constant relative risk aversion $(\leq 1)$, zero opportunity cost, and zero initial wealth. Our result generalizes his result to an $n$-person, non-limiting case.

The theorem shows that at each stage the anchor player $P_{0}$ and the non-anchor player $P_{i}$ splits what is remaining of the net surplus $\left\{\pi-\left(T_{0}+\sum_{j=1}^{n} T_{j}\right)\right\} \prod_{j=1}^{i-1} r_{j}$ in the ratio $r_{i}: 1-r_{i}$. In the symmetric case where all players have the same intertemporal preferences, we have

\section{Corollary 1 Suppose that there exists some $\rho$ such that}

$$
\rho_{i}=\rho \text { for } i=0,1, \ldots, n \text {. }
$$

Then the equilibrium payoffs are

$$
x_{0}=\left(1-\tau_{0}\right)\left(\pi-\sum_{j=0}^{n} T_{j}\right) \frac{1}{(1+\rho)^{n}}
$$

and

$$
x_{i}=\left(1-\tau_{i}\right)\left(\pi-\sum_{j=0}^{n} T_{j}\right) \frac{\rho}{(1+\rho)^{i}} \text { for } i=1, \ldots, n \text {. }
$$

The corollary says that in the symmetric case, at each stage, the anchor player $P_{0}$ and the non-anchor player $P_{i}$ split what is remaining of the net surplus $(\pi-$ $\left.\sum_{j=0}^{n} T_{j}\right) \frac{1}{(1+\rho)^{i-1}}$ in the ratio $1: \rho$.

Corollary 2 Suppose that there are no third-party transfers: $T_{0}=T_{1}=\cdots=T_{n}=0$, $\tau_{0}=\tau_{1}=\cdots=\tau_{n}=0$. In the limiting case where $\rho \rightarrow 1$, one has 


$$
x_{0}=\frac{1}{2^{n}} \pi
$$

and

$$
x_{i}=\frac{1}{2^{i}} \pi \text { for } i=1, \ldots, n
$$

In the limiting symmetric case where impatience disappears, $P_{1}$ takes one half of the pie, and each one after $P_{1}$ also takes one half of what is remaining of the pie. The last non-anchor player $P_{n}$ and the anchor player $P_{0}$ each take one half of what is left of the pie at the last stage. The following fairy-tale story may provide some intuition for the result:

"A boy wants to get to treasures in a secret garden. In order to reach the secret garden, the boy has to go through a series of gates. At each gate, a monster demands a share of the treasures in return for letting the boy through. The boy and the monster have the same degrees of impatience so that the boy has to promise to yield one half of what is remaining of the treasures to the monster to get through the gate."

The boy in the story will end up with only a tiny fraction of treasures after giving one half of the current amount of treasures to the gate-keeping monster at each gate. The most advantageous position is that of the monster at the first gate.

\section{Concluding remarks}

The bargaining protocol introduced in this paper can be used in a bargaining situation where one player faces hierarchical opponents to negotiate with. The core nature of the protocol is that the anchor negotiator can move to the next negotiation only after completing the current negotiation. We proved that there exists a unique perfect equilibrium of the game under certain assumptions on the intertemporal preferences of the players. In particular, we assumed that the players' preferences are such that the present value is linear in the size of the stake.

With third-party transfers, we showed that lump-sum transfers are jointly shared by all players, while a proportional transfer only affects the player who is obliged to make the transfer. With only proportional transfers and zero lump-sum transfers, an anchor player and each non-anchor player bargain as if there is no further bargaining.

We also demonstrated that with the hierarchical anchored bargaining protocol we introduced, the anchor player and the last non-anchor player are in the most disadvantageous position, while the first non-anchor player is in the most advantageous position. Obviously, in this kind of a situation, if players have any choice, they would like to become the first non-anchor player rather than an anchor player. Given that many real-life multiple-party bargaining procedures use a fixed mediator and sequential bargaining, resembling the protocol in the current paper, there is some explanation to do. Within the framework of our model, one explanation could be that there is a 
large subsidy $(T<0)$ for an anchor player so that the anchor player has an incentive to secure agreements from other parties even though the anchor player has to share his subsidy with other players. There may be, however, other explanations not based on our model. Analyzing alternative models would be useful in broadening our understanding of the prevalence of sequential bargaining procedures with a fixed mediator.

In the current work, we dealt with a pure bargaining situation, where there is one fixed pie that could be split among all players only with a unanimous agreement. One extension would be to endogenize the pie to be split between the anchor player and a non-anchor player at each stage of the bargaining game. In such a model, one could investigate the efficiency of allocations with sequential bargaining.

Acknowledgements We would like to thank an anonymous referee and associate editor for thoughtful and helpful comments. Of course, any errors and shortcomings are our own.

Open Access This article is distributed under the terms of the Creative Commons Attribution 4.0 International License (http://creativecommons.org/licenses/by/4.0/), which permits unrestricted use, distribution, and reproduction in any medium, provided you give appropriate credit to the original author(s) and the source, provide a link to the Creative Commons license, and indicate if changes were made.

\section{Appendix}

Proposition 1 A preference relation $\succeq$ over $(x, t) \in \mathbb{R}_{+}^{2}$ that satisfies axioms $A 1-A 5$ and $A 6^{*}$ can be represented by a utility function $u(x, t)=\delta^{t} u(x)$ where $0<\delta<1$ and $u(x)=x^{\alpha}$ for some $\alpha>0$.

Proof By Fishburn and Rubinstein (1982), a preference relation $\succeq$ over $(x, t)$ that satisfies axioms A1-A5 can be represented by $u(x, t)=\delta^{t} u(x)$ where $0<\delta<1$ and $u(x)$ is a continuous and increasing real-valued function. If $\mathrm{A} 6 *$ is satisfied, one has

$$
u\left(\rho^{t} x\right)=\delta^{t} u(x) .
$$

Without loss of generality, we may assume $u(1)=1$. Then one has for $y \geq 0$

$$
u\left(\rho^{y}\right)=\delta^{y} .
$$

For $y<0$, let $y=-t$ for $t>0$. Then

$$
1=u(1)=u\left(\rho^{t} \rho^{y}\right)=\delta^{t} u\left(\rho^{y}\right)
$$

and thus

$$
u\left(\rho^{y}\right)=\delta^{-t}=\delta^{y} .
$$

We have shown that for any $y \in \mathbb{R}$

$$
u\left(\rho^{y}\right)=\delta^{y} .
$$

Now, let $\rho^{y}=x$, then $u(x)=x^{\alpha}$ where $\alpha=\log _{\rho} \delta$. 


\section{References}

Bharath S, Panchapegesan V, Werner I (2014) The changing nature of chapter 11, Indian Institute of Management Bangalore WP No: 461

Cai H (2000) Delay in multilateral bargaining under complete information. J Econ Theory 93:260-276

Chae S (2002) Tax incidence with bargaining. Econ Lett 77:199-204

Chae S, Yang J (1988) The unique perfect equilibrium of an n-person bargaining game. Econ Lett 28:221223

Chae S, Yang J (1992) An n-person bargaining process with alternating demands. Seoul J Econ 3:255-261

Chae S, Yang J (1994) An n-person pure bargaining game. J Econ Theory 62:86-102

Coase R (1960) The problem of social cost. J Law Econ 3:1-44

Fishburn PC, Rubinstein A (1982) Time preference. Int Econ Rev 23:677-694

Genicot G, Ray D (2006) Contracts and externalities: how things fall apart. J Econ Theory 131:71-100

Herrero M (1985) A strategic bargaining approach to market institutions, Ph.D. Thesis. London School of Economics, London

Iaryczower M, Oliveros S (2017) Competing for loyalty: the dynamics of rallying support. Am Econ Rev 107:2990-3005

Jun BH (1987) A structural consideration on 3-person bargaining, Ph.D. Thesis. University of Pennsylvania, Philadelphia

Krishna V, Serrano R (1996) Multilateral bargaining. Rev Econ Stud 63:61-80

Lee J, Liu Q (2013) Gambling reputation: repeated bargaining with outside options. Econometrica 81:16011672

Nash JF (1950) The bargaining problem. Econometrica 18:155-162

Nash JF (1953) Two-person cooperative games. Econometrica 21:128-140

Rubinstein A (1982) Perfect equilibrium in a bargaining model. Econometrica 50:97-109

Sutton J (1986) Non-cooperative bargaining theory: an introduction. Rev Econ Stud 53:709-724

Xiao J (2018) Bargaining orders in a multi-person bargaining game. Games Econ Behav 107:364-379

Publisher's Note Springer Nature remains neutral with regard to jurisdictional claims in published maps and institutional affiliations. 